\title{
Wide-field fundus autofluorescence corresponds to visual fields in chorioretinitis patients
}

This article was published in the following Dove Press journal:

Clinical Ophthalmology

28 November 2011

Number of times this article has been viewed

\author{
Florian Seidensticker ${ }^{1}$ \\ Aljoscha S Neubauer' \\ Tamer Wasfy ${ }^{1,2}$ \\ Carmen Stumpf' \\ Stephan R Thurau' ${ }^{1, *}$ \\ Anselm Kampik' \\ Marcus Kernt ${ }^{1, *}$ \\ 'Department of Ophthalmology, \\ Ludwig-Maximilians-University, \\ Munich, Germany; ${ }^{2}$ Department \\ of Ophthalmology, Tanta \\ University, Tanta, Egypt \\ *Both authors contributed equally \\ to this work
}

Correspondence: Marcus Kernt Department of Ophthalmology, LudwigMaximilians-University Munich, Mathildenstr. 8, 80336 Munich, Germany Tel +4989516038II

Fax +498951605160

Email marcus.kernt@med.uni-muenchen.de
Background and objectives: Detection of peripheral fundus autofluorescence (FAF) using conventional scanning laser ophthalmoscopes (SLOs) is difficult and requires pupil dilation. Here we evaluated the diagnostic properties of wide-field FAF detected by a two-laser wavelength wide-field SLO in uveitis patients.

Study design/materials and methods: Observational case series of four patients suffering from different types of posterior uveitis/chorioretinitis. Wide-field FAF images were compared to visual fields. Panretinal FAF was detected by a newly developed SLO, which allows FAF imaging of up to $200^{\circ}$ of the retina in one scan without the need for pupil dilation. Visual fields were obtained by Goldmann manual perimetry.

Results: Findings from wide-field FAF imaging showed correspondence to visual field defects in all cases.

Conclusion: Wide-field FAF allowed the detection of visual field defect-related alterations of the retinal pigment epithelium in all four uveitis cases.

Keywords: fundus autofluorescence (FAF), Optomap, wide-field scanning laser ophthalmoscopy, imaging, uveitis, visual field

\section{Introduction}

Detection of peripheral fundus autofluorescence (FAF) using conventional scanning laser ophthalmoscopes (SLOs) is often difficult, as most of these imaging devices only allow $30^{\circ}$ to $60^{\circ}$ of the retina to be captured in one scan. In addition, pupil dilation is usually required for adequate image quality. ${ }^{1}$

Recently, the nonmydriatic two-laser wavelength wide-field SLO Optomap (Optomap P200Tx; Optos PLC, Dunfermline, Fife, Scotland) has been developed. This novel device allows detection of FAF for up to $200^{\circ}$ of the retina in one scan. Therefore, the Optomap P200Tx provides nonmydriatic imaging not only for the posterior pole of the retina, but even extending over the equator. ${ }^{2}$

The Optomap P200Tx SLO includes two scanning laser wavelengths: green $(532 \mathrm{~nm})$ and red $(633 \mathrm{~nm})$. For detection of FAF, the green laser is sent into the eye and used as an exciter. Certain fluorophores in the eye (eg, lipofuscin) that emit light in a range from $\sim 540$ to $>700 \mathrm{~nm}$ are excited and autofluorescence can be detected. The FAF signal is mainly derived from lipofuscin and its dominant fluorophore $N$-retinylidene- $N$-retinyl-ethanolamine (A2E), accumulated in the retinal pigment epithelium (RPE). ${ }^{3}$ The Optomap imaging device differs from other FAF detection devices, as it uses a green laser for excitation and detects retinal autofluorescence within the yellow-orange-red range. One potential advantage of using the 
532-nm wavelength for excitation is that the FAF signal may be more specific for lipofuscin, as interference from collagen autofluorescence may be reduced, compared with excitation of FAF at lower wavelengths (eg, $488 \mathrm{~nm}$, as used in the Heidelberg HRA 2 FAF detection system [Heidelberg Engineering, Heidelberg, Germany]). ${ }^{1,3,4}$ The emitted FAF signal of the retina is detected from a raster scan and a bright-band detector for 570 to $780 \mathrm{~nm}$. In addition to the detection of wide-field FAF, the Optomap P200Tx allows viewing of the two separate images from the red and green laser scans or both scans superimposed to yield semirealistic color imaging. For both fundus imaging and wide-field FAF, only a small optical pass of $\geq 2 \mathrm{~mm}$ is required and the specific mirror design of the Optomap P200AF prototype allows wide-field images of approximately $180^{\circ}-200^{\circ}$ to be obtained without pupil dilation. $^{2}$ The optical resolution of the specific instrument used in this study was $3900 \times 3072$ pixels, resulting in approximately 17-22 pixels per degree. The acquisition time was 0.25 seconds per scan.

Here we report our initial experience with Optomap wide-field FAF images in four patients with chorioretinitis.

\section{Case I}

A 62-year-old man with human leukocyte antigen serotype A29 (HLA-A29)-positive birdshot chorioretinopathy and a history of blurred vision in both eyes came for a control visit to our uveitis clinic. Best-corrected visual acuity (BCVA) of the right and left eyes was 20/20 and 20/25. The intraocular pressure (IOP) was $15 \mathrm{mmHg}$ in the right eye and $17 \mathrm{mmHg}$ in the left eye. Slit-lamp examination revealed minimal anterior segment inflammation. Fundus examination of both eyes showed cream-colored and depigmented spots (Figure 1A and B). Visual fields were measured by Goldmann manual perimetry (scotoma right eye: temporal $35^{\circ}$, nasal $5^{\circ}$, superior $30^{\circ}$, inferior $35^{\circ}$, left eye: temporal $55^{\circ}$, nasal $35^{\circ}$, superior $35^{\circ}$, inferior $0^{\circ}$ ). The visual fields revealed paracentral scotoma in the upper temporal area in the right eye and an arcuate scotoma extending from the blind spot nasally in the left eye. A full clinical examination and Optomap FAF imaging were performed. Areas of reduced FAF corresponded to the visual field defects (Figure $1 \mathrm{C}-\mathrm{F}$ ). In the right eye, the defect was $\mathrm{C}$-shaped around the macula and the peripapillary region, extending temporally. The left eye showed a partial loss of autofluorescence centrally and absolute confluent defects below the macula. Areas of reduced FAF corresponded markedly with visual field losses and choroidal damage.
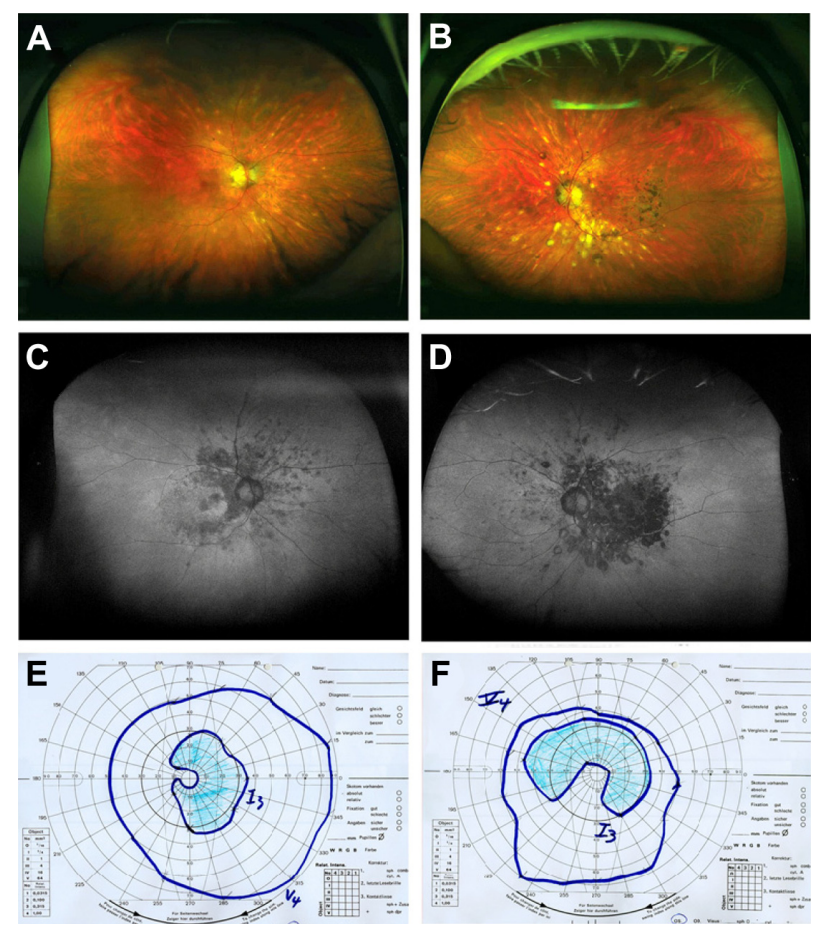

Figure I Case I: (A, B, C, and D) Optomap color fundus imaging (A and B) and wide-field FAF ( $\mathbf{C}$ and $\mathbf{D}$ ) of the right $(\mathbf{A}$ and $\mathbf{C}$ ) and left eye (B and $\mathbf{D})$. ( $(\mathbf{E}$ and $\mathbf{F}$ ) corresponding visual fields measured with Goldmann manual perimetry. The nasal paracentral scotoma of the right eye, and the large scotoma extending from the blind spot nasally on the left eye correspond well with the areas of reduced FAF. Abbreviation: FAF, fundus autofluorescence.

\section{Case 2}

A 48-year-old man suffering from visual loss due to Vogt-Koyanagi-Harada syndrome in both eyes came for a follow-up visit to our uveitis clinic. He was under oral cyclosporine treatment (200 mg/day) and had pars plana vitrectomy with silicone oil due to exudative retinal detachment on his right eye one year prior to the visit. The left eye had been treated intravitreally with triamcinolone (4 mg) 19 months prior. On examination, the visual acuity was count fingers on the right eye and 20/100 on the left eye. Routine examination revealed an IOP of $15 \mathrm{mmHg}$ in the right and $18 \mathrm{mmHg}$ in the left. Slit-lamp examination of the anterior chamber and vitreous of both eyes was normal. Fundus examination of the right eye revealed a whitish central lesion under silicone oil. The left eye showed multiple, deep creamy-yellow choroidal lesions at the posterior pole and an old paracentral scar (Figure 2A). The visual field loss of the left eye showed a scotoma in the superior field sparing the macula. Corresponding to the visual field (scotoma left eye: temporal $20^{\circ}$, nasal $15^{\circ}$, superior $15^{\circ}$, inferior $0^{\circ}$ ), a loss of FAF was seen extending from the papilla below and temporal to the macula (Figure 2B and $\mathrm{C}$ ). 

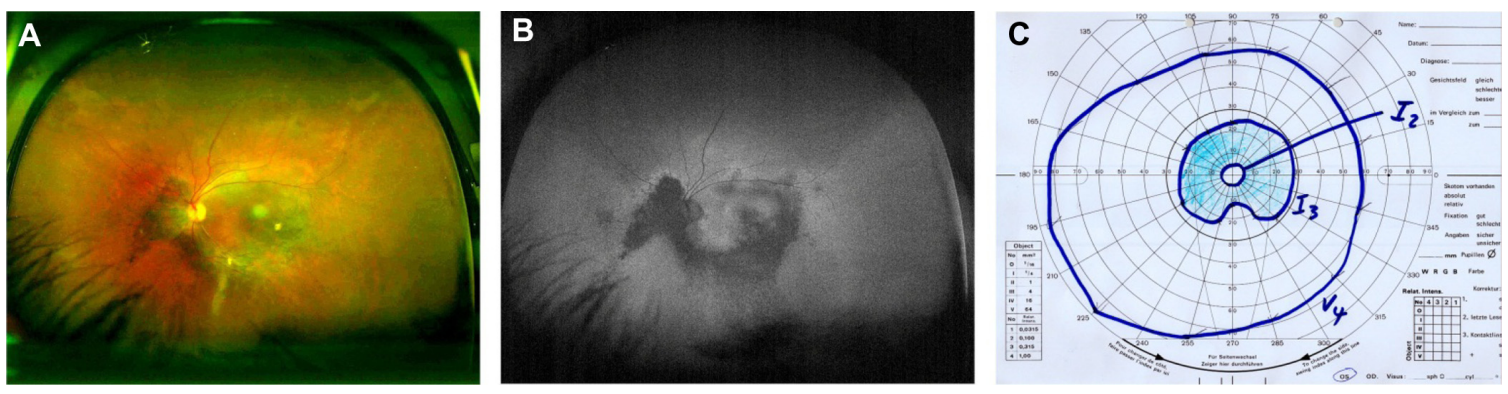

Figure 2 Case 2: (A and B) Optomap color fundus imaging $(\mathbf{A})$ and wide-field FAF (B) of the right eye. (C) Corresponding visual field measured with Goldmann manual perimetry.

Abbreviation: FAF, fundus autofluorescence.

\section{Case 3}

A 52-year-old patient was diagnosed with Vogt-KoyanagiHarada syndrome five years ago and was under systemic infliximab treatment $(5 \mathrm{mg} / \mathrm{kg})$ for three months. At the time of presentation, the patient reported a change in visual acuity after several weeks. His BCVA was finger count in the right eye and 20/400 in the left eye. The IOP in both eyes was $13 \mathrm{mmHg}$. Slit-lamp examination showed a bilateral anterior granulomatous uveitis with keratic precipitates and few cells and flare in the anterior chamber's aqueous fluid. Fundus examination of both eyes revealed moderate vitritis, choroidal folds, macular thickening, and perivascular edema. FAF revealed peripapillary arc-shaped areas of hypofluorescence extending from the central pole, surrounded by hyperfluorescent borders (Figure $3 \mathrm{C}$ and D). Findings from FAF, especially regarding the hypofluorescence areas, showed correspondence to relative scotoma in visual field obtained by Goldmann perimetry (scotoma right eye: temporal $20^{\circ}$, nasal $20^{\circ}$, superior $20^{\circ}$, inferior $0^{\circ}$, left eye: temporal $20^{\circ}$, nasal $25^{\circ}$, superior $20^{\circ}$, inferior $20^{\circ}$ ) (Figure 3).

\section{Case 4}

A 64-year-old man presented with complaints of decreased visual acuity in the right eye for four days secondary to a relapse of his long-standing history of choroiditis. At the time of presentation, no medication was taken. Visual acuity of the right and left eyes was 20/200 and 10/200. The IOP was $10 \mathrm{mmHg}$ in the right eye and $12 \mathrm{mmHg}$ in the left eye. Both eyes showed normal anterior chamber findings on slitlamp examination. Multiple old cells were seen in the vitreous. Fundus examination on both eyes showed pigmented scars in the area of the posterior pole and pigmented scars in the periphery with no signs of active inflammation (Figure 4A and B). Visual field testing on both eyes revealed central and nasal scotoma corresponding to the defects noted upon FAF examination (scotoma right eye: temporal $20^{\circ}$, nasal $30^{\circ}$, superior $30^{\circ}$, inferior $5^{\circ}$, left eye: temporal $15^{\circ}$, nasal $30^{\circ}$, superior $20^{\circ}$, inferior $5^{\circ}$ ). The autofluorescence images showed a distinctive geographic configuration that originated peripapillary and in the macular area and extended centrifugally (Figure 4C and D). The areas of RPE atrophy corresponded to the visual field as measured by Goldmann perimetry, presenting a central scotoma and decreased peripheral field of vision (Figure 4C-F).

\section{Discussion}

FAF has proven to be a valuable tool for the detection of RPE pathologies in several diseases. FAF has been used for
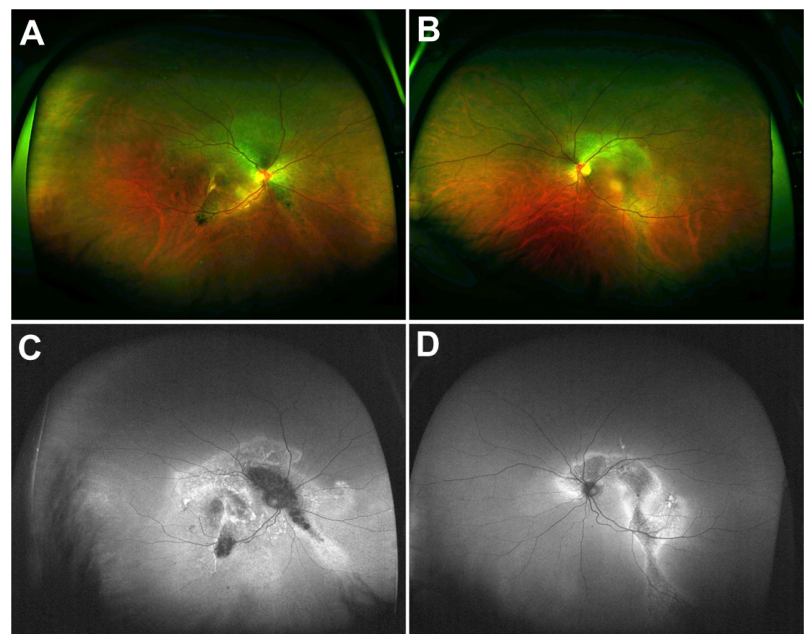

$\mathbf{E}$

$\mathbf{F}$

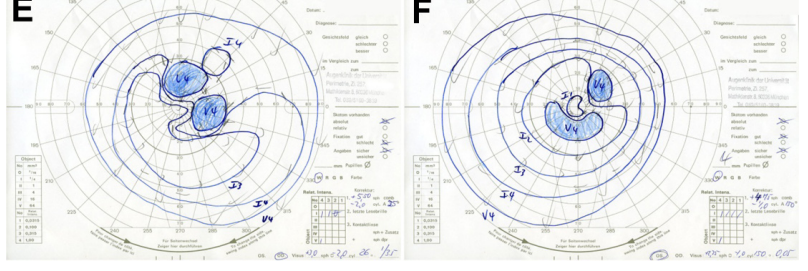

Figure 3 Case 3: (A, B, C, and D) Optomap color imaging (A and B), wide-field FAF ( $\mathbf{C}$ and $\mathbf{D}$ ) of the right and left eye, and corresponding visual fields ( $\mathbf{E}$ and $\mathbf{F})$. In both eyes peripapillary arc-shaped areas of hypofluorescence extending from the central pole, surrounded by hyperfluorescent borders can be seen in Optomap FAF ( $C$ and $\mathbf{D}$ ). Abbreviation: FAF, fundus autofluorescence. 

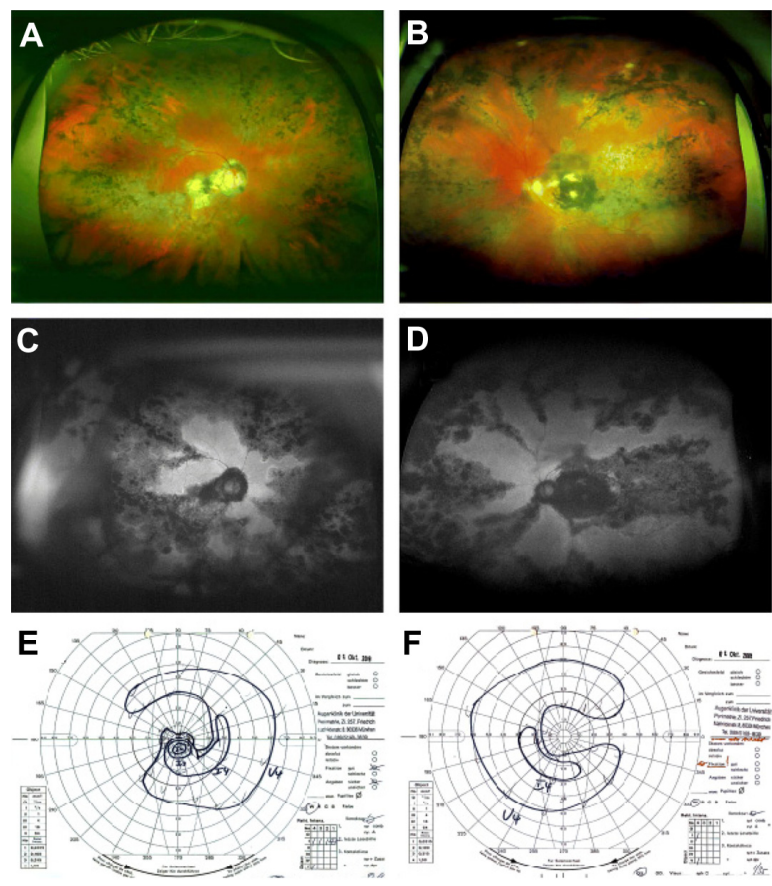

Figure 4 Case 4: (A, B, C, and D) Optomap color imaging (A and B), wide-field FAF ( $\mathbf{C}$ and $\mathbf{D})$ of the right eye and left, and corresponding visual fields (E and $\mathbf{F}$ ). In both eyes, pigmented scars at the posterior pole and the periphery can be seen in Optomap color fundus imaging. FAF reveals distinct serpiginous and geographic areas of reduced FAF in both eyes. These findings correspond to visual field defects found in Goldmann perimetry.

Abbreviation: FAF, fundus autofluorescence.

both detection and monitoring of geographic atrophy secondary to age-related macular degeneration, diabetic maculopathy, and hereditary retinal disorders such as Stargardt disease. ${ }^{1,5-7}$ In addition, FAF has been shown to be an appropriate technique to detect inflammatory damage in chorioretinitis patients. ${ }^{7-9}$ Especially in patients with agerelated macular degeneration, irregularities in central FAF have been correlated with a decrease in visual acuity. ${ }^{1}$ However, peripheral changes in the RPE often occur as well, potentially resulting in visual field defects. Detection of FAF using conventional imaging devices is usually limited to the posterior pole, as only $30^{\circ}$ to $60^{\circ}$ of the retina can be imaged in one scan. ${ }^{1}$ Therefore, only limited data regarding peripheral FAF and potential correlations to visual field defects are available.

The Optomap imaging system allows detection of FAF for up to $200^{\circ}$ by using a green laser $(532 \mathrm{~nm})$ for excitation of FAF and a broad-band detector within the yellow-orangered range. ${ }^{2}$ These features potentially result in more specific detection of lipofuscin-associated FAF, as interference from collagen autofluorescence may be less than when using lower excitation wavelengths. ${ }^{1,3,4}$ These unique properties of the Optomap P200Tx system allow better detection of FAF related to peripheral RPE alterations and may provide additional information regarding RPE-related retinal dysfunction of the peripheral retina.

Here we describe the use of this wide-field FAF imaging technique in four patients with chorioretinitis. Birdshot chorioretinopathy, and Vogt-Koyanagi-Harada syndrome are clinically well-characterized uveitis entities resulting in atrophic damage to the choriocapillaris, pigment epithelium, and adjacent outer layers of the retina, and specific changes in FAF have been described. ${ }^{8,9}$ This damage causes focal loss of visual acuity in the affected areas. Previous studies have investigated autofluorescence of retinal pigment at infrared wavelengths as well as wavelengths in the blue (excitation) and green (emission) spectra. Using the Optomap P200Tx, we investigated the autofluorescence with a green light excitation at $532 \mathrm{~nm}$ and emission at 570 to $780 \mathrm{~nm}$. We found deep defects of autofluorescence in the damaged retinal areas. The four patients with focal loss of autofluorescence underwent visual field testing, which showed deep scotomas. Comparing the images from FAF detection with the visual fields, we found high concordance.

In the presented four cases, changes in FAF are closely related to certain inflammatory retinal changes. Hyperfluorescent areas in FAF have been described mainly as a result of hyperplastic pigment epithelium, whereas hypofluorescent areas seem to be closely correlated to decreased RPE activity and atrophy for example as a result of scarring from chronic or previous inflammation. Studies have demonstrated in many other diseases that loss of autofluorescence might indicate loss of function, which sometimes is irreversible. ${ }^{1,3,6,9,10}$ Findings from these four patients indicate that the Optomap P200Tx prototype might detect defective autofluorescence corresponding closely with loss of function. If this holds true in further studies, an additional tool is available that can detect damage and possibly new areas of damage after relapses. Wide-field FAF imaging potentially provides a noninvasive technique to monitor areas of old or new retinal inflammatory activity and may provide additional information to slit-lamp examination or visual field examination alone. An additional advantage of the Optomap P200Tx is that only a minimal optical pass ( $\geq 2 \mathrm{~mm}$ ) is required. Therefore, wide-field FAF imaging can be obtained quick and easily even in eyes with undilated pupils. In addition, due to the SLO principle, the Optomap P200Tx provides good quality images even in eyes with media opacities due to intraocular inflammation.

\section{Disclosure}

The authors report no conflicts of interest in this work. 


\section{References}

1. Schmitz-Valckenberg S, Bültmann S, Dreyhaupt J, Bindewald A, Holz FG, Rohrschneider K. Fundus autofluorescence and fundus perimetry in the junctional zone of geographic atrophy in patients with age-related macular degeneration. Invest Ophthalmol Vis Sci. 2004; 45(12):4470-4476.

2. Kernt M, Schaller UC, Stumpf C, Ulbig MW, Kampik A, Neubauer AS. Choroidal pigmented lesions imaged by ultra-wide-field scanning laser ophthalmoscopy with two laser wavelengths (Optomap). Clin Ophthalmol. 2010;4:829-836.

3. Hammer M, Königsdörffer E, Liebermann C, et al. Ocular fundus autofluorescence observations at different wavelengths in patients with age-related macular degeneration and diabetic retinopathy. Graefes Arch Clin Exp Ophthalmol. 2008;246(1):105-114.

4. Schweitzer D, Schenke S, Hammer M, et al. Towards metabolic mapping of the human retina. Microsc Res Tech. 2007;70(5):410-419.

5. Boon CJ, Jeroen Klevering B, Keunen JE, Hoyng CB, Theelen T. Fundus autofluorescence imaging of retinal dystrophies. Vision Res. 2008;48(26): 2569-2577.
6. Fleckenstein M, Charbel Issa P, Fuchs HA, et al. Discrete arcs of increased fundus autofluorescence in retinal dystrophies and functional correlate on microperimetry. Eye (Lond). 2009;23(3):567-575.

7. Giuliari G, Hinkle DM, Foster CS. The spectrum of fundus autofluorescence findings in birdshot chorioretinopathy. J Ophthalmol. 2009; 2009:567693.

8. Vasconcelos-Santos DV, Sohn EH, Sadda S, Rao NA. Retinal pigment epithelial changes in chronic Vogt-Koyanagi-Harada disease: fundus autofluorescence and spectral domain-optical coherence tomography findings. Retina. 2010;30(1):33-41.

9. Yeh S, Forooghian F, Wong WT, et al. Fundus autofluorescence imaging of the white dot syndromes. Arch Ophthalmol. 2010;128(1):46-56.

10. Vujosevic S, Casciano M, Pilotto E, Boccassini B, Varano M, Midena E. Diabetic macular edema: fundus autofluorescence and functional correlations. Invest Ophthalmol Vis Sci. 2011;52(1):442-448.
Clinical Ophthalmology

\section{Publish your work in this journal}

Clinical Ophthalmology is an international, peer-reviewed journal covering all subspecialties within ophthalmology. Key topics include: Optometry; Visual science; Pharmacology and drug therapy in eye diseases; Basic Sciences; Primary and Secondary eye care; Patient Safety and Quality of Care Improvements. This journal is indexed on

Submit your manuscript here: http://www.dovepress.com/clinical-ophthalmology-journal

\section{Dovepress}

PubMed Central and CAS, and is the official journal of The Society of Clinical Ophthalmology (SCO). The manuscript management system is completely online and includes a very quick and fair peer-review system, which is all easy to use. Visit http://www.dovepress.com/ testimonials.php to read real quotes from published authors. 